International Journal of Applied Mathematical Research, 1 (4) (2012) 487-492 (C)Science Publishing Corporation

www.sciencepubco.com/index.php/IJAMR

\title{
k-trees, k-ctrees and Line Splitting Graphs
}

\author{
Veena Mathad* and B.Basavanagoud** \\ *DOS in Mathematics, University of Mysore, Manasagangotri, \\ Mysore-570 006, Karnataka State, India. \\ Email:veena_mathad@rediffmail.com \\ **Department of Mathematics, Karnatak University, \\ Dharwad-580 003, Karnataka State, India. \\ Email:b.basavanagoud@gmail.com
}

\begin{abstract}
Let $G=(V, E)$ be a graph. For each edge $e_{i}$ of $G$, a new vertex $e_{i}^{\prime}$ is taken and the resulting set of vertices is denoted by $E_{1}(G)$. The line splitting graph $L_{s}(G)$ of a graph $G$ is defined as the graph having vertex set $E(G) \cup E_{1}(G)$ with two vertices adjacent if they correspond to adjacent edges of $G$ or one corresponds to an element $e_{i}^{\prime}$ of $E_{1}(G)$ and the other to an element $e_{j}$ of $E(G)$ where $e_{j}$ is in $N\left(e_{i}\right)$. In this paper we characterize graphs whose line splitting graphs are $k$-trees and $k$-ctrees.
\end{abstract}

Keywords: $k$-trees, $k$-ctrees, line splitting graph, splitting graph.

\section{Introduction}

By a graph $G=(V, E)$, we mean a finite, undirected graph without loops or multiple edges. For graph theoretic terminology, we refer to [1].

A vertex $v$ of a graph $G$ is called a star-vertex if all its neighboring vertices are independent.

A graph $G$ is said to be $n$-degenerate if every subgraph of $G$ has a vertex of degree atmost $n$ [3]. 
The open neighborhood $N(u)$ of a vertex $u$ in $V(G)$ is the set of vertices adjacent to $u$. For each vertex $u_{i}$ of $G$, a new vertex $u_{i}^{\prime}$ is taken and the resulting set of vertices is denoted by $V_{1}(G)$.

The splitting graph $S(G)$ of a graph $G$ is defined as the graph having vertex set $V(G) \cup V_{1}(G)$ with two vertices adjacent if they correspond to adjacent vertices of $G$ or one corresponds to an element $u_{i}^{\prime}$ of $V_{1}(G)$ and the other to an element $w_{j}$ of $V(G)$ where $w_{j}$ is in $N\left(u_{i}\right)[7]$

The open neighborhood $N\left(e_{i}\right)$ of an edge $e_{i}$ in $E(G)$ is the set of edges adjacent to $e_{i}$. For each edge $e_{i}$ of $G$, a new vertex $e_{i}^{\prime}$ is taken and the resulting set of vertices is denoted by $E_{1}(G)$.

The line splitting graph $L_{s}(G)$ of a graph $G$ is defined as the graph having vertex set $E(G) \cup E_{1}(G)$ with two vertices adjacent if they correspond to adjacent edges of $G$ or one corresponds to an element $e_{i}^{\prime}$ of $E_{1}(G)$ and the other to an element $e_{j}$ of $E(G)$ where $e_{j}$ is in $N\left(e_{i}\right)$ [2].

Remark 1.1. If $G=L(H)$ for some graph $H$, then $G=S(L(H))$.

The simplest way to define a $k$-tree for $k \geq 1$ is by recursion. A $k$-tree of order $k+1$ is a complete graph of order $k+1$. A $k$-tree of order $p+1, p \geq k+1$, can be obtained by joining a new vertex to any $k$ mutually adjacent vertices of a $k$-tree of order $p$. Note that 1 -trees are generally known as 'trees' [4].

Remark 1.2. [6] Every 2 - tree is planar.

The class of $k$-ctrees (for $k \geq 1$ ) is the set of all graphs that can be obtained by the following recursive construction rule.

1. A totally disconnected graph of order $k$ (i.e., $\overline{K_{k}}$ ) is a $k$-ctree.

2. To a $k$-ctree $Q^{\prime}$ of order $n-1$ (where $n>k$ ), insert a new $n^{\text {th }}$ vertex and join it to any set of $k$ independent vertices of $Q^{\prime}$.

Note that 1 -ctrees are generally known as trees [5].

Theorem 1.3. [2] The line splitting graph $L_{s}(G)$ of a graph $G$ is planar if and only if $G$ is planar and $(i)$ or $($ ii) holds :

1. $G$ is either $K_{1,4}$ or $C_{2 n}, n \geq 2$.

2. $\triangle(G) \leq 3$ and $G$ has no subgraph homeomorphic from the subdivision graph of $K_{1,3}$ and also every block of $G$ is either a $K_{2}$ or a triangle such that each triangle has atmost one cut-vertex. 
Theorem 1.4. [4] Let $G$ be a graph of order $p$ and let $k<p$. Then the following assertions are equivalent :

1. $G$ is a $k$-tree.

2. $G$ is $k$-connected, triangulated and $K_{k+2}-$ free.

3. $G$ is $k$-connected, triangulated of size $k p-\left(\begin{array}{c}k+1 \\ 2\end{array}\right)$

Theorem 1.5. [1] If $G$ is a $(p, q)$ graph whose vertices have degree $d_{i}$, then the line graph of $G, L(G)$ has $q$ vertices and $Q_{L}$ edges, where $q_{L}=-q+\frac{1}{2} \sum d_{i}^{2}$.

Theorem 1.6. [5] Let $G$ be a graph of order $p \geq 2 k$. Then $G$ is $k$-ctree if and only if $G$ is a $k$-degenerate, triangle-free graph of size $k(p-k)$.

Theorem 1.7. [5] A graph $G$ of order $\geq k+1$ is a $k$-ctree if and only if $G$ has a star-vertex $v$ of degree $k$ and $G-v$ is a $k$-ctree.

Theorem 1.8. [5] Every $k$-ctree is a $k$-degenerate, triangle-free graph.

\section{Main Results}

\section{$2.1 \quad \mathrm{k}$-trees and Line splitting graphs}

Theorem 2.1. There are only two graphs whose line splitting graphs are 2 -trees. These graphs are $K_{1,3}$ and $C_{3}$.

Proof. Suppose the line splitting graph $L_{s}(G)$ of a graph $G$ is a $2-$ tree. Clearly $G$ is connected. By Remark 1.2, $L_{s}(G)$ is planar and hence by Theorem 1.3, $G$ is planar and is either $K_{1,4}$ or $C_{2 n}, n \geq 2$ or $\triangle(G) \leq 3$ and $G$ has no subgraph homeomorphic from the subdivision graph of $K_{1,3}$ and also every block of $G$ is either a $K_{2}$ or a triangle such that each triangle has atmost one cut-vertex. We consider the following cases depending on the magnitude of $\triangle(G)$.

Case 1. $\triangle(G)=1$. Then $G=K_{2}$. Clearly, $L_{s}(G)$ is disconnected, a contradiction.

Case 2. $\triangle(G)=2$. Then $G$ is either a path or a cycle. Let $G$ be a graph of order $p$ and size $q$. We have the following subcases in this case.

Subcase 2.1. $G$ is a path $P_{p}, p \geq 3$. For $p=3, G=K_{1,2}$. But $L_{s}\left(K_{1,2}\right)$ is not triangulated and hence by Theorem $1.4, L_{s}(G)$ is not a 2 -tree, a contradiction. For $p \geq 4, L_{s}(G)$ contains a cycle of length $n=4$ without chords and therefore, $L_{s}(G)$ is not triangulated, a contradiction.

Subcase 2.2. $G$ is a cycle $C_{p}, p \geq 3$. Then $L_{s}(G)$ has $2 p$ vertices and $3 p$ edges. Since a 2 -tree with $2 p$ vertices contains $4 p-3$ edges, it follows that $3 p<4 p-3$ for all $p>3$ and $3 p=4 p-3$ for $p=3$. Hence $G=C_{3}$.

Case 3. $\triangle(G)=3$. We consider the following subcases. 
Subcase 3.1. $G$ is not a tree. Then $G$ has atleast one cycle. So $G$ is a cycle together with a path of length $\geq 1$, adjoined at some vertex. Then $L_{s}(G)$ contains a cycle of length $n=4$ without chords and therefore, $L_{s}(G)$ is not triangulated, a contradiction.

Subcase 3.2. $G$ is a tree other than $K_{1,3}$. Then $L_{s}(G)$ contains a cut-vertex and by Theorem 1.4, $L_{s}(G)$ is not a 2 -tree, a contradiction. Hence $G=K_{1,3}$. Case 4. $\triangle(G)>3$. Then $K_{1,4}$ is a subgraph of $G$. One can see that $L_{s}\left(K_{1,4}\right)$ contains a subgraph isomorphic to $K_{4}$ and therefore $L_{s}(G)$ is not a 2 -tree, a contradiction.

From all the above cases, it follows that $G=K_{1,3}$ or $C_{3}$.

Theorem 2.2. A line splitting graph $L_{s}(G)$ of order $2(k+1), k \geq 3$, is a $k$-tree if and only if $G=K_{1, k+1}$.

Proof. Let $G$ be a $(p, q)$ graph. It follows from Theorem 1.5, that the line graph $L(G)$ is $\left(q,-q+\frac{1}{2} \sum d_{i}^{2}\right)$ graph, where $d_{i}$ is degree of each vertex $v_{i} \in V(G)$. Suppose that $L_{s}(G)$ is a $k-$ tree, $k \geq 3$ of order $2(k+1)$. Then clearly $p_{L}=q=k+1$. Also, since $L_{s}(G)=S(L(G)), L_{s}(G)$ contains $3\left(-q+\frac{1}{2} \sum d_{i}^{2}\right)$ edges. Since $L_{s}(G)$ is a $k$-tree, we have,

$$
\begin{aligned}
\left|E\left(L_{s}(G)\right)\right| & =2(k+1) k-\frac{k(k+1)}{2} \\
& =\frac{3 k(k+1)}{2} .
\end{aligned}
$$

This implies that

$$
\begin{aligned}
3\left(-q+\frac{1}{2} \sum d_{i}^{2}\right) & =\frac{3 k(k+1)}{2} . \\
\text { So, } \quad q_{L} & =\frac{k(k+1)}{2} .
\end{aligned}
$$

Therefore, $L(G)=K_{k+1}, k \geq 3$ and hence $G$ is $K_{1, k+1}, k \geq 3$.

Conversely, suppose that $K_{1, k+1}, k \geq 3$. Then $L(G)=K_{k+1}$. Hence $L_{s}(G)=S(L(G))$ is a $k$-tree of order $2(k+1), k \geq 3$.

\section{$2.2 \quad$ k-ctrees and Line splitting graphs}

Theorem 2.3. There is only one graph whose the line splitting graph is 1 -ctree. That graph is $P_{3}$.

Proof. Suppose the line splitting graph of a graph $G$ is a $1-c t r e e$. Then $L_{s}(G)$ is a tree. Assume that $G$ has a vertex $u$ of $d e g \geq 3$. Then any three edges incident with $u$ form $K_{1,3}$. Consequently, $L_{s}(G)$ contains a triangle $K_{3}$. This 
is impossible since $L_{s}(G)$ is a tree. Hence $\triangle(G) \leq 2$. Then every component of $G$ is either a cycle $C_{n}, n \geq 3$ or a path $P_{n}, n \geq 2$. If $G$ is a cycle $C_{n}$, then $L_{s}(G)$ contains a subgraph $C_{n}$, which is impossible since $L_{s}(G)$ is a tree. So, $G$ must be a path. Now, if the length of the path is 1 , then $L_{s}(G)$ is $2 K_{1}$, which is not 1 -ctree. Also, if the length of the path is $\geq 3$ then $L_{s}(G)$ contains a cycle $C_{4}$, which is impossible. Hence $G=P_{3}$.

Theorem 2.4. There are only two graphs whose the line splitting graphs are $2-$ ctrees. These graphs are $K_{2}$ and $C_{4}$.

Proof. Suppose that the line splitting graph of a graph $G(p, q)$ is a 2 -ctree. Suppose $p \geq 5$. We consider the following cases.

Case 1. $\triangle(G) \geq 3$. Then $L_{s}(G)$ is not triangle-free. By Theorem 1.6, $L_{s}(G)$ is not a $2-$ ctree, a contradiction.

Case 2. $\triangle(G) \leq 2$. Then $G$ is either a cycle $C_{p}, p \geq 5$ or a path of length atleast 4 . We consider the following subcases :

Subcase 2.1. $G=C_{p}, p \geq 5$, then $L_{s}(G)$ contains $2 p$ vertices and $3 p$ edges. But by Theorem 1.6, a 2 -ctree on $2 p$ vertices has $4 p-4$ edges. Since $p \geq 5$ we have $3 p<4 p-4$, a contradiction.

Subcase 2.2. $G=P_{p}, p \geq 5$, then $L_{s}(G)$ contains $2 p-2$ vertices and $3 p-6$ edges. But by Theorem 1.6, a 2 -ctree on $2 p-2$ vertices has $4 p-8$ edges. Since $p \geq 5$ we have $3 p-4<4 p-8$, a contradiction.

In all the cases we arrive at a contradiction. Thus $p \leq 4$. In this case, $L_{s}(G)$ is isomorphic to one of the graphs, $\overline{K_{2}}$ and $G_{1}$, where $G_{1}$ is a graph shown in Figure 1. Consequently $G$ is $K_{2}$ and $C_{4}$, respectively.

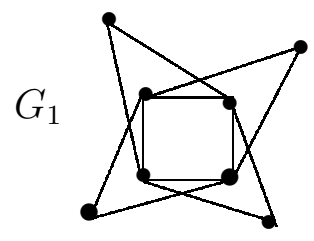

Figure 1.

Theorem 2.5. $L_{s}(G)$ is a $k$-ctree, $k \geq 3$ if and only if $k=2 l, l \geq 2$ and $G=l K_{2}$.

Proof. Suppose that $L_{s}(G)$ is a $k$-ctree, $k \geq 3$ of order $p$ for some graph $G$. It follows from Theorem 1.8 that $L_{s}(G)$ is triangle-free graph. Assume that $p \geq k+1$. Then by Theorem $1.7, L_{s}(G)$ contains a star-vertex $u$ of degree $k$ and $L_{s}(G)-u$ is a $k$-ctree. We consider the following cases:

Case 1. $u$ corresponds to a newly introduced vertex $e^{\prime}$ for an edge $e$ of $G$. Then $e$ is adjacent to $k$ edges in $G$. Since $k \geq 3, L_{s}(G)$ contains a triangle, a contradiction.

Case 2. $u$ corresponds to an edge $e$ of $G$. By construction of $L_{s}(G), e$ is 
adjacent to $\frac{k}{2}$ edges in $G$. Since number of edges is an integer, $k$ must be even. Also since $k \geq 3$, we have $k=2 l, l \geq 2$. So, $e$ is adjacent to atleast two edges in $G$. We consider the following subcases :

Subcase 2.1. $e$ is adjacent to more than two edges in $G$, then $L_{s}(G)$ contains a triangle, a contradiction.

Subcase 2.2. $e$ is adjacent to exactly two edges at its same end vertices in $G$, then also $L_{s}(G)$ contains a triangle, a contradiction.

Subcase 2.3. $e$ is adjacent to exactly two edges at its different end vertices in $G$, then $L_{s}(G)-e$ contains either a pendant vertex or an isolated vertex. Since $k \geq 3$, it follows that $L_{s}(G)-e$ is not a $k$-ctree, a contradiction.

In all the cases we arrive at a contradiction. So $p=k$. Hence $L_{s}(G)$ is a $k$-ctree of order $k$. It follows that $L_{s}(G)=\bar{K}_{k}$. Now, if $k=2 l+1, l \geq 1$, then $L_{s}(G)$ has odd number of vertices, which is impossible. Hence, $k=2 l, l \geq 2$ and $G=l K_{2}$.

Conversely, suppose $G=l K_{2}, l \geq 2$. Then $L(G)=l K_{1}$ and hence $L_{s}(G)$ is $2 l K_{1}$. i.e. $k K_{1}$, which is a $k-$ ctree of order $k=2 l, l \geq 2$. i.e. $k \geq 3$.

Corollary 2.6. There are no graphs whose line splitting graphs are $k-$ ctrees where $k=2 l+1, l \geq 1$.

\section{References}

[1] F. Harary, Graph Theory, Addison Wesley, 1969.

[2] V.R.Kulli and M.S.Biradar, The line splitting graph of a graph, Acta Ciencia Indica, 28 (M.No.3) (2002), pp.317-322.

[3] D.R.Lick and A.T.White, $k$-degenerate graphs, Canad.J.Math., 22 (1970), pp.1082-1096.

[4] H.P.Patil, On the Structure of $k$-trees, J.Comb., Inform and System Sci., 11(No.2-4) (1986), pp.57-64.

[5] H.P.Patil and R.Pandiyaraj, On the structure of $k$-ctrees and their applications to graph valued functions, Proceedings of the National Conference on Graph Theory and Its Applications, Tumkur University, Tumkur, (Dec 5th 2011), pp.1-8.

[6] H.P.Patil(Warsaw), $k$ - trees and some graph valued functions, Discussiones Mathematicae, 7 (1985), pp.87-92.

[7] E.Sampathkumar and H.B.Walikar, On splitting graph of a graph, J.Karnatak University Sci. 25 and 26(combined) (1980-81), pp.13-16. 